\title{
Do Radiological Measurements Correspond to Functional Outcome in Fractures of Distal Radius?- A Cross Sectional Study
}

\author{
Charles Abraham¹, Nithin Thomas Philip², Grace Mary John³ \\ ${ }^{1}$ Department of Orthopaedics, Believers Church Medical College, Thiruvalla, Pathanamthitta, Kerala, India. \\ ${ }^{2}$ Department of Orthopaedics, Believers Church Medical College, Thiruvalla, Pathanamthitta, Kerala, India. \\ ${ }^{3}$ Department of Clinical Pharmacy, Believers Church Medical College, Thiruvalla, Pathanamthitta, Kerala, India.
}

\section{ABSTRACT}

\section{BACKGROUND}

One in six fractures seen in the emergency department is a distal radius fracture and is by far the most common fracture of the upper extremity. The relationship between functional outcome and radiological parameters following distal radius fractures remains controversial. Several studies have shown that functional outcome does not always correspond with radiological perfection, and vice versa. There are only few studies showing the relative importance of each radiological factor in the influence of functional outcomes. We wanted to determine the relationship between the radiological parameters and functional outcome of distal radius fracture.

\section{METHODS}

This is a cross sectional analytical study conducted using data obtained from patient records and radiological examination of patients who were treated in our medical college for distal radius fracture between February 2018 and February 2019. There were 44 patients satisfying the inclusion criteria who had distal radius fractures treated both conservatively and surgically (closed K- wire fixation or volar plating). Cases in which true PA and lateral X-ray were unavailable and cases for which sufficient data at six months follow up was unavailable were excluded from the study. All patients underwent the standard physiotherapy protocol. Post-operative radial height, radial angulation and palmar tilt, were measured by 3 independent observers and the mean value along with DASH score was obtained from patients at 6 months. These parameters were analysed for relationship using Welch t-test for significance.

\section{RESULTS}

The mean age of patients in our study was $44 \pm 15 \mathrm{yrs}$. The male to female ratio was found to be 2.64. The Radial height was measured 6 months post treatment and divided into two groups $(>10 \mathrm{~mm}$ and $<10 \mathrm{~mm}$ ) which was associated with an average DASH score of $3.6 \pm 5.1$ and 3.6 \pm 5.6 . Using the Welch t-test, the association of the Radial height with DASH score was evaluated and was found to be not significant $\left(\mathrm{p}\right.$ value $=1.0$ ). Likewise the Radial inclination $>15^{\circ}$ was associated with an average DASH score of $4.3 \pm 5.5$ and for $<15^{\circ}$ correction, the average DASH score was $0 \pm 0$ which was statistically significant ( $\mathrm{p}$ value $=0.0001$ ) and for volar tilt $<13^{\circ}$ was associated with a lower DASH score of $2.7 \pm 4.3$ when compared with a volar tilt $>13^{\circ}$ which had a DASH score of $6.8 \pm 6.8$ which was found to be statistically significant ( $\mathrm{p}$ value $=0.025$ )

\section{CONCLUSIONS}

Our study was able to demonstrate a statistically significant relation between the radial inclination and volar tilt with the DASH score, but the radial height did not show a statistically significantly relation with the DASH score.

\section{KEY WORDS}

Distal Radius Fracture, Functional Outcome, Radiological Outcome, Radial Height, Volar Tilt, Radial Inclination.
Corresponding Author: Dr. Nithin Thomas Philip, Department of Orthopaedics, Believers Church Medical College, Thiruvalla, Pathanamthitta, Kerala, India. E-mail: drnithintphilip@gmail.com

DOI: $10.14260 / \mathrm{jemds} / 2019 / 816$

Financial or Other Competing Interests: None.

How to Cite This Article: Abraham C, Philip NT, John GM. Do radiological measurements correspond to functional outcome in fractures of distal radius? a cross sectional study. J. Evolution Med. Dent. Sci. 2019;8(50):3769-3771, DOI: 10.14260/jemds/2019/816

Submission 14-10-2019, Peer Review 30-11-2019, Acceptance 06-12-2019, Published 16-12-2019. 


\section{BACKGROUND}

One in six fractures seen in emergency department is a distal radius fracture and is by far the most common fracture of the upper extremity. ${ }^{1}$ Vast majority of the patient are in the elderly age group with multiple co-morbidities making them not a suitable candidate for surgical fixation. They also have low functional demands which warrant conservative management with closed reduction and cast application. Due to the lack of definitive guidelines, it is often difficult to treat a patient with complex articular involvement. Restoring the articular congruity as the key factor in treatment of such facture was projected by several studies.2,3,4 Cooney et al ${ }^{5}$ also concluded in his study that restoration of the articular surface with open reduction and internal fixation will reduce the likelihood of loss of reduction and attain better joint congruity than with closed treatment. We often see patients without any functional problems despite grossly displaced healed distal radius fractures. The functional and radiological relationship following distal radius fractures remains controversial. Several studies have shown that functional outcome does not always correspond with radiological perfection, $6,7,8$ and vice versa. 9,10,11 Radial Height, Volar tilt and Radial inclination are the most commonly used radiographic parameters predicting the functional outcome after surgery. However, there have been controversies concerning the relative importance of each factor in the influence of functional outcomes. Tsukazaki et $\mathrm{al}^{8}$ showed only Volar tilt corresponded with functional outcome but Batra et al ${ }^{11}$ study showed radial height was the radiographic parameter most strongly affecting the functional outcome. Hence this study was conducted to find out the relation between the individual radiological parameters and functional outcome of distal radius fractures.

\section{METHODS}

A Cross-sectional analytical study design was implemented using data obtained from patient records and radiological examinations of patients who were treated in our medical college for distal radius fracture from February 2018 to February 2019. We included patients with distal radius fractures treated both conservatively and surgically. Surgical treatment involved closed K-wire fixation ${ }^{12,13}$ or Volar plating. ${ }^{14}$ There were 44 patients satisfying the inclusion criteria and all of them were selected for the study without sampling. Exclusion criteria were those patients who did not have a true PA or lateral X-ray of the wrist and insufficient data at 6 months follow-up. All patients underwent the standard physiotherapy protocol. Post-operative radial height, radial inclination and volar tilt were measured by 3 independent observers in pre-treatment and 6 months post treatment Xray. All radiographic measurement acquisition was from a standardized PA and lateral views of the wrist and the mean value calculated by three independent observers were taken for comparison. To subjectively assess the patient's functional abilities, the self-reported measures of disabilities of the arm, shoulder and hand (DASH) questionnaire ${ }^{15}$ was used, and the mean value was found at 6 months follow up. The Welch t-test was used to find out the statistical significance of each radiological parameter in the functional outcome as indicated by the DASH score.

\section{RESULTS}

\section{A. Demographics}

Out of the 44 , majority were males $(68.18 \%, \mathrm{n}=30)$ and females comprised of nearly $31.81 \%(n=14)$. Age group of patients ranged from 19 years to 73 years, with an average age of 46 years. The largest number of patients fell in the age group of 50 - 59 years $(25 \%, \mathrm{n}=11)$. Out of the 44 patients, $52.27 \%$ patients had left distal radius fracture $(n=23)$ and $47.73 \%$ patient had right sided injury $(\mathrm{n}=21)$.

\begin{tabular}{|c|c|c|}
\hline Age Group & No. of Patients & Percentage (\%) \\
\hline $10-19$ & 1 & 2.3 \\
\hline $20-29$ & 10 & 22.7 \\
\hline $30-39$ & 5 & 11.4 \\
\hline $40-49$ & 7 & 15.9 \\
\hline $50-59$ & 11 & 25.0 \\
\hline $60-69$ & 8 & 18.2 \\
\hline $70-79$ & 2 & 4.5 \\
\hline Total & $\mathbf{4 4}$ & $\mathbf{1 0 0}$ \\
\hline \multicolumn{3}{|c|}{ Age Group } \\
\hline
\end{tabular}

\begin{tabular}{|c|c|c|}
\hline Gender & No. of Patients & Percentage (\%) \\
\hline Female & 14 & 31.81 \\
\hline Male & 30 & 68.18 \\
\hline \multicolumn{2}{|c|}{ Gender } \\
\hline
\end{tabular}

\begin{tabular}{|c|c|c|}
\hline Side of Injury & No. of Patients & Percentage (\%) \\
\hline Right & 21 & 47.73 \\
\hline Left & 23 & 52.27 \\
\hline \multicolumn{3}{|c|}{ Side of Injury } \\
\hline
\end{tabular}

\section{B. Radial Height}

The Radial height post treatment was divided into two groups $(>10 \mathrm{~mm}$ and $<10 \mathrm{~mm})$. The $>10 \mathrm{~mm}(\mathrm{n}=25)$ group and $<10$ $\mathrm{mm}(\mathrm{n}=17)$ group was associated with an average DASH score of $3.6 \pm 5.1$ and $3.6 \pm 5.6$. Using the Welch $\mathrm{t}$-test the association of the Radial height with DASH score was evaluated and was found to be not significant. The power of this study result was calculated and found to be only $5 \%$.
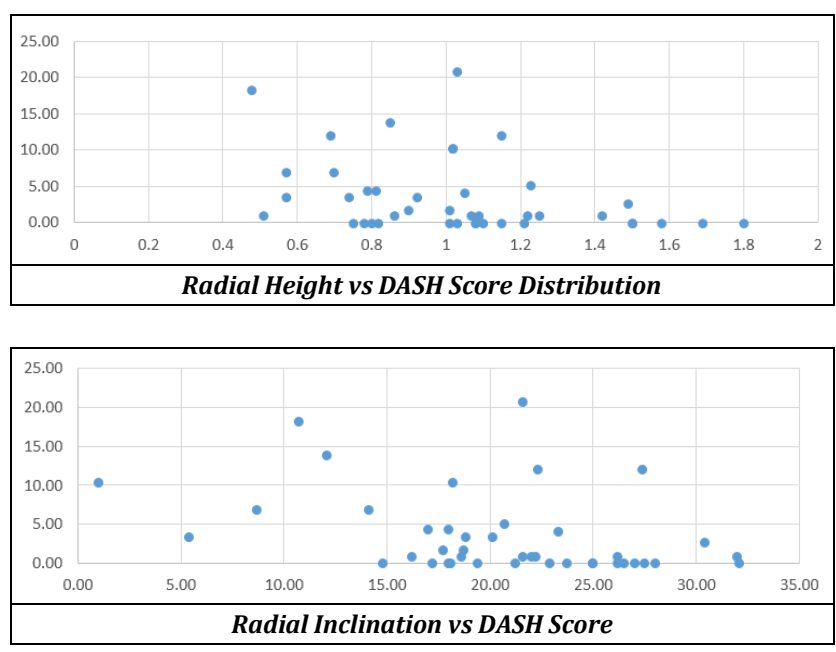

\section{Radial Inclination}

The radial inclination measurements were divided into two groups $\left(>15^{\circ}\right.$ and $\left.<15^{\circ}\right)$. The $>15^{0}$ group $(n=35)$ was 
associated with an average DASH score of $4.3 \pm 5.5$ and for $<15^{\circ}(\mathrm{n}-=7)$ had an average DASH score was $0 \pm 0$. Using the Weltch $\mathrm{t}$ test, the relationship of radial inclination and DASH score was evaluated and was found to be significant. ( $p$ value $=0.0001$ ).

\section{Volar Tilt}

The volar tilt measured to be in the $<13$ group $(n=35)$ was associated with a lower DASH score of $2.7 \pm 4.3$ when compared with a volar tilt $>13^{\circ}(\mathrm{n}=9)$ which had a DASH score of $6.8 \pm 6.8$. Using the Weltch t-test the association of volar tilt with DASH score was evaluated and was found to be significant. $(\mathrm{p}$ value $=0.025)$.

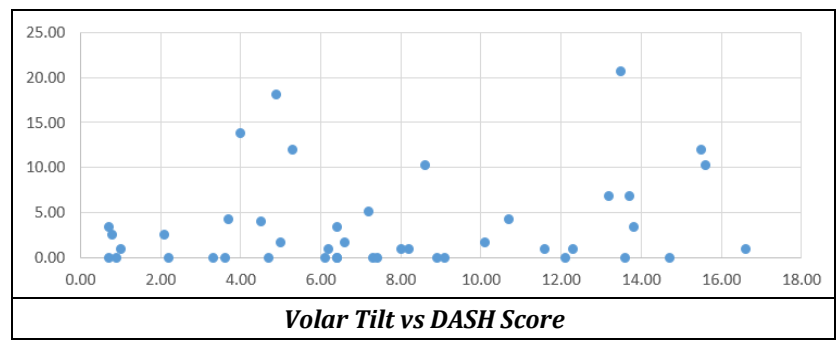

\section{DISCUSSION}

In our study we found that the DASH score was significantly lower in those patients whose radial inclination and volar tilt were corrected towards the normal limit however the radial height did not show a significant association with the functional results. Several studies (Gartland and Werley 1951 and Solgaard 1989) suggested radial height as a weak predictor of functional outcome but they were short term studies and was not statistically supported. Studies by Sarmiento et al (1975), Villar et al (1987) and Yen et al for 3year period proposed radial height as the radiological parameter suggesting functional outcome but in our study we were not able to define a significant association of radial height with that of the functional outcome which may be attributed to the short term follow-up of 6 months. We also calculated the power of that study and was found to be only $5 \%$ which signifies that a larger cohort is needed to suggest its relation.

\section{CONCLUSIONS}

Short-term functional outcome depends mainly on the correction of the volar tilt and the radial inclination to its normal anatomical state. Though the radial height did not correspond to the functional outcome in our study a definitive conclusion cannot be made. This may be due to the short follow-up of 6 months and the power calculated was only $5 \%$ for radial height which shows that a larger sample size is need for a definitive conclusion. Hence, we conclude that the restoration of the Radial inclination and volar tilt to near normal is essential for a better short-term functional outcome in the treatment of distal radius fracture. Longer duration follow-up with larger cohort is needed to further establish its significance.

\section{REFERENCES}

[1] Chen NC, Jupiter JB. Management of distal radial fractures. J Bone Joint Surg Am 2007;89(9):2051-62.

[2] Axelrod TS, McMurtry RY. Open reduction and internal fixation of comminuted. Intra-articular fractures of the distal radius. J Hand Surg Am 1990;15(1):1-I I.

[3] Bradway JK. Amadio PC, Cooney WP. Open reduction and internal fixation of displaced, comminuted intra-articular fractures of the distal end of the radius. J Bone Joint Surg 1989;71(6):839-47.

[4] Fernandez DL, Geissler WB. Treatment of displaced articular fracture of the radius. J Hand Surg Am 1991;16(3):375-84.

[5] Cooney WP 3rd, Linscheid RL, Dobyns JH. External pin fixation for unstable Colle's fractures. J Bone Joint Surg Am 1979;61(6A):840-5.

[6] Jaremko JL, Lambert RG, Rowe BH, et al. Do radiographic indices of distal radius fracture reduction predict outcomes in older adults receiving conservative treatment? Clin Radiol 2007;62(1):65-72.

[7] Anzarut A, Johnson JA, Rowe BH, et al. Radiologic and patient-reported functional outcomes in an elderly cohort with conservatively treated distal radius fractures. J Hand Surg Am 2004;29(6):1121-7.

[8] Tsukazaki T, Takagi K, Iwasaki K. Poor correlation between functional results and radiographic findings in Colles' fracture. J Hand Surg Br 1993;18(5):588-91.

[9] Fujii K, Henmi T, Kanematsu Y, et al. Fractures of the distal end of radius in elderly patients: a comparative study of anatomical and functional results. J Orthop Surg 2002;10(1):9-15.

[10] Karnezis IA, Fragkiadakis EG. Association between objective clinical variables and patient-rated disability of the wrist. J Bone Joint Surg Br 2002;84(7):967-70.

[11] Batra S, Gupta A. The effect of fracture-related factors on the functional outcome at 1 year in distal radius fractures. Injury 2002;33(6):499-502.

[12] Lichtman DM, Bindra RR, Boyer MI, et al. Treatment of distal radius fractures. J Am Acad Orthop Surg 2010;18(3):180-9.

[13] Philip NT, Abraham C. A prospective study on functional, clinical and radiological evaluation of distal radius fracture treated with five percutaneous k-wire. Medpulse International Journal of Orthopedics 2019;11(2):46-9.

[14] Laino DK, Tejwani N. Indications for operative fixation of distal radius fractures: a review of the evidence. Bull NYU Hosp Jt Dis 2012;70(1):35-40.

[15] Hudak PL, Amadio PC, Bombardier C. Development of an upper extremity outcome measure: the DASH (disabilities of the arm, shoulder and hand) [corrected]. The Upper Extremity Collaborative Group (UECG). Am J Ind Med 1996;29(6):602-8. 